Société d'histoire de la révolution de 1848 et des

révolutions du XIXe siècle

$50 \mid 2015$

Sociétés et forces de sécurité au XIXe siècle

\title{
Police et société dans l'Allemagne impériale : la chasse au criminel dans la métropole berlinoise
}

Police and society in imperial Germany: Hunting down criminals in Berlin metropolis

Polizei und Gesellschaft im deutschen Kaiserreich: Die Jagd auf Kriminelle in Berlin

\section{Philipp Müller}

Traducteur : Nicolas Le Moigne

\section{Q OpenEdition Journals}

Édition électronique

URL : http://journals.openedition.org/rh19/4822

DOI : $10.4000 /$ rh 19.4822

ISSN : $1777-5329$

Éditeur

La Société de 1848

Édition imprimée

Date de publication : 1 juillet 2015

Pagination : 79-97

ISSN : 1265-1354

Référence électronique

Philipp Müller, « Police et société dans l'Allemagne impériale : la chasse au criminel dans la métropole berlinoise », Revue d'histoire du XIXe siècle [En ligne], 50 | 2015, mis en ligne le 01 juillet 2015, consulté le 20 avril 2019. URL : http://journals.openedition.org/rh19/4822 ; DOI : 10.4000/rh19.4822 


\section{PHILIPP MÜLLER}

\section{Police et société dans l'Allemagne impériale : la chasse au criminel dans la métropole berlinoise1}

Le 15 juin 1904, deux écoliers berlinois retrouvent, à proximité du pont de Beussel sur le canal de Spandau, la tête et les bras d'un enfant. Le lendemain, le Berliner Lokal-Anzeiger rapporte que «les deux enfants ont immédiatement pensé que leur trouvaille avait peut-être à voir avec le meurtre de Lucie Berlin $»^{2}$. Leur découverte donne lieu, en tout cas, à un reportage précis et circonstancié3 ; quatre jours auparavant, le tronc de la jeune fille disparue avait déjà été retrouvé et évoqué dans un article du journal concurrent, le Berliner Morgenpost, qui l'avait fait afficher à divers endroits de la ville . $^{4}$ La découverte des morceaux manquants du cadavre ne se fait pas attendre, à mesure que l'esprit public s'échauffe au sujet de l'affaire. Le 17 juin, un employé d'une entreprise de charbon a «tout de suite des soupçons» lorsqu'il voit près du canal «un objet emballé dans un tissu rouge et ficelé avec des fils de laine» - des passants retrouvent finalement, le même jour, les membres manquants du corps dans la Spree'.

Le meurtre de Lucie Berlin fait vite les gorges chaudes de la capitale, ainsi que la «une» des principaux organes de presse durant une dizaine de jours ${ }^{6}$. Le Berliner Lokal-Anzeiger suit l'affaire semaine après semaine; le Berliner Beobachter estime de son côté que l'affaire Lucie Berlin s'est emparée des esprits, toutes classes sociales confondues, de l'ouvrier buveur de bière au bourgeois amateur de Sekt, des habitants des immeubles avec pignon sur rue à ceux des arrière-cours ${ }^{7}$. Partout où l'on en parle, cette affaire "sensationnelle», suscite, d'après le Berliner Beobachter, «l'expression de la commi-

1. L'article a été traduit par Nicolas Le Moigne. Je remercie par ailleurs le comité éditorial et les rapporteurs pour leurs remarques et leurs conseils.

2. BLA (Berliner Lokal-Anzeiger), 16.6.1904, $\mathrm{n}^{\circ} 272$, p. 3 ; BMP (Berliner Morgenpost), 16.8.1904, $\mathrm{n}^{\circ} 270$, p. 1.

3. BLA 16.6.1904, $\mathrm{n}^{\circ} 277$, p. 3 ; BT (Berliner Tageblatt), 16.6.1904, $\mathrm{n}^{\circ} 301$, supplément, p. 2.

4. $B L A, 11.6 .1904, \mathrm{n}^{\circ} 270$, p. $1 ; B M P, 12.6 .1904, \mathrm{n}^{\circ} 136$, supplément, p. 1.

5. $B L A, 17.6 .1904, \mathrm{n}^{\circ} 280$, p. $2 ; B T, 17.6 .1904, \mathrm{n}^{\circ} 304$, p. $4 ; B M P, 18.6 .1904, \mathrm{n}^{\circ} 141$, supplément, p. 2.

6. BLA, 19.6.1904, $\mathrm{n}^{\circ} 283$, supplément, p. 1.

7. Cf. Rosemarie Beier, «Leben in der Mietskaserne. Zum Alltag Berliner Unterschichtfamilien in den Jahren 1900 bis 1920 ", in Gesine Asmus [dir.], Hinterhof, Keller Mansarde, Einblicke ins Berliner Wohnungselend, Reinbeck, Rowohlt, 1982, p. 244-269. 
sération, de la compassion, des propos bons et humains $»^{8}-$ et les dons des voisins montrent que le public se sent concerné. La chronique du Berliner Lokal-Anzeiger garde davantage de distances vis-à-vis des aspects les plus sensationnels de l'affaire, marquant un écart sensible avec les habitants de la métropole. Cet article se penche sur la question des affaires criminelles sensationnelles à Berlin durant l'époque impériale, et sur leurs dynamiques dans l'espace public ${ }^{9}$ : 'affaire Lucie Berlin, mais aussi les affaires Wetzel en 1891, ainsi que l'affaire Grosse et Werner en 1896. Les sources principales sont d'une part les archives du ministère prussien de l'Intérieur et de la préfecture de police de Berlin ${ }^{10}$, et d'autre part les rapports de la police criminelle reproduits dans les principaux journaux de la presse berlinoise ${ }^{11}$. L'analyse de cette documentation hétérogène suivra un axe prioritaire : la place du crime drama dans la communication publique ${ }^{12}$, afin de mettre en lumière l'action concertée de la police, de la presse et de la population pour élucider les différents cas et appréhender les criminels.

Une première piste de réflexion consiste à se poser la question des rapports d'intérêts de la police et des organes de presse, de leurs modes d'action spécifiques et de leur coopération institutionnelle. La seconde est la façon dont la population reçoit les informations et met en œuvre, dans une grande métropole, les appels à la coopération lancés par les institutions. Enfin, il nous faut retrouver la trace des sentiments du public et de ses réactions, reflétés et commentés par les journaux. La thèse centrale de cette mise en perspective est de montrer que, pour le plus grand nombre, la recherche des criminels dans la ville donnait le sentiment d'une participation à la vie publique plus importante que celle que permettaient les institutions et la vie politique encore censitaires et exclusivement masculines ${ }^{13}$. La collusion officieuse entre la presse quotidienne et la police, de son côté, a fait évoluer le rapport entre la police et la société, à travers la collaboration des "civils» aux enquêtes, à la chasse

8. $B L A, 19.6 .1904, \mathrm{n}^{\circ} 283$, supplément, p. 1.

9. Cette réflexion prolonge des travaux déjà publiés : Philipp Müller, Auf der Suche nach dem Täter. Die öffentliche Dramatisierung von Verbrechen im Berlin des Kaiserreichs, Frankfurt-am-Main, Campus, 2005; «Éducateur ou mauvais garçon? Le capitaine de Köpenick et les bouleversements du paysage médiatique dans l'Allemagne de Guillaume II", in Jörg Requate [dir.], Das 19. Jahrhundert als Mediengesellschaft, München, 2009, p. 89-98; "Covering Crime, Restoring Order. The 'Berlin Jack the Ripper' and the Press Policy of the Berlin Criminal Investigation Department", Crime, Histoire \& Sociétés, vol. 15, 2011-1, p. 85-110.

10. Cf. également Albrecht Funk, Polizei und Rechtsstaat. Die Entwicklung des staatlichen Gewaltmonopols in Preußen 1848-1918, Frankfurt-am-Main, Campus, 1986.

11. Les fonds d'archives sont néanmoins très lacunaires, en raison des destructions subies durant la Seconde Guerre mondiale par le Geheimes Staatsarchiv PK (GStA PK) et par le Landesarchiv Berlin (LAB). Les archives des éditeurs de journaux ont quant à elles été détruites. L'historien doit donc dans certains cas compenser ces manques par les sources imprimées, quant à elles abondantes. Les rapports de police sont cependant en partie disponibles dans la mesure où ils sont repris et diffusés par la presse à partir de 1884. Sur les sources, $c f$. en particulier Philipp Müller, Auf der Suche nach dem Täter, op. cit., p. 30 et $s q$.

12. Sur la notion de crime drama, cf. Eric A. Johnson, Urbanization and Crime, Germany 18711914, Cambridge, CUP, 1995, p. 2.

13. Michael Erbe, "Berlin im Kaiserreich (1871-1918)», in Wofgang Ribbe [dir.], Geschichte Berlins, vol. 2, Munich, Beck, 1987, p. 688-744. 
aux criminels sous la forme d'avis de recherche ou parfois de poursuites spontanées dans la rue : au bout du compte, l'affaire criminelle se transforme en spectacle social. Les nouvelles possibilités offertes aux masses ont été l'objet de controverses, car elles posaient la question du rôle public des individus et de leur légitimité sociale à un moment où l'Empire connaissait de profonds bouleversements sociaux et culturels. Savoir qui devait participer à ces chasses aux criminels, et de quelle manière, revenait toujours à poser la question du rôle public de l'individu et des attentes sociales qui l'entourent en termes de comportement - et également la question implicite du pouvoir, celle de savoir qui a le droit d'apparaitre, de s'exprimer dans l'espace public, et de quelle manière. Les chasses aux criminels à Berlin, et l'imprégnation policière grandissante de la société, prennent racine dans les tensions politiques et culturelles qui marquent l'époque de l'Empire ${ }^{14}$. Autrement dit, les «nouveaux lecteurs" consomment les journaux locaux à bon marché et les communiqués de presse de la police ${ }^{15}$, se repaissent de l'opportunisme avec lequel ces journaux utilisent les enquêtes policières, et s'opposent par là-même aux «lecteurs établis».

Inscrite dans le sillage ouvert par Judith Walkowitz dans son City of Dreadful Delight, au sujet des différences sociales dans la perception des discours criminologiques dans la presse anglaise ${ }^{16}$, ainsi que dans celui des travaux de Dominique Kalifa sur les liens entre crime et société en France au XIX ${ }^{e}$ siècle ${ }^{17}$, notre étude entend également poursuivre les éléments de réflexion apportés par Peter Fritzsche, dans Reading Berlin, sur le journal en tant que "ciment médiatique de la ville de Berlin»" ${ }^{18}$, en insistant notamment sur les mécanismes d'appropriation concrète des discours médiatiques ${ }^{19}$. Le paramètre historique à prendre en compte pour comprendre le succès de ce média métropolitain est qu'un journal est davantage une chose lue qu'une chose imprimée. Les informations et les idées qu'il véhicule circulent ${ }^{20}$.

14. Cf. Benjamin C. Hett, Death in the Tiergarten. Murder and Criminal Justice in the Kaiser's Berlin, Cambridge (Mass.), HUP, 2004.

15. Martin Lyons, "Die neuen Leser im 19. Jahrhundert. Frauen, Kinder, Arbeiter», in Roger Chartier et Gulielmo Cavallo [dir.], Die Welt des Lesens, Frankfurt-am-Main, Campus, 1999, p. 455497.

16. Judith Walkowitz, City of Dreadful Delight. Narratives of Sexual Danger in Late-Victorian London, London, 1992.

17. Dominique Kalifa, L'encre et le sang. Récits de crimes et société à la Belle Époque, Paris, Fayard, 1995; Crime et culture au XIX'e siècle, Paris, Perrin, 2005.

18. Peter Fritzsche, Reading Berlin, Cambridge (Mass.), HUP, 1996.

19. Philipp Müller, "Journalistische Vermittlung und ihre Aneignung. Öffentliche Verhandlungen über den Fall Wilhelm Vogt in Berlin 1906/1908", Österreichische Zeitschrift für Geschichtswissenschaften, 13, 2002, vol. 2, p. 35-56; Suche nach dem Täter, op. cit.; Peter Becker, Dem Täter auf der Spur. Poliziearbeit und Kriminalistik im 19. Jahrhundert, Köln, Wiss. Buchges, 2005; Sace Elder, «Murder, Denunication and criminal policing in Weimar Berlin ", Journal of Contemporay History, $\mathrm{n}^{\circ}$ 41, 2006, p. 401-419; Murder Scenes, Normality, Deviance, and Criminal Violence in Weimar Berlin, Ann Arbor, University of Michigan Press, 2010; Rebekka Habermas, "Von Anselm von Feuerbach zu Jack the Ripper. Recht und Kriminalität im 19. Jahrhundert. Ein Literaturbericht ", Rechtsgeschichte, 3, 2003, p. 128-261.

20. Le sociologue Robert E. Park rappelle dans ses analyses sur la presse moderne qu'il ne s'agirait 
Analyser comment les "nouveaux lecteurs" se sont appropriés les enquêtes criminelles s'impose de façon d'autant plus forte que les lecteurs engagés sont souvent pris à témoin dans les articles comme étant «le public». Â cet égard, il est frappant qu'un singulier collectif classe souvent la chose dont il est question dans la catégorie des entités passives, qui consomment plus qu'elles ne créent. Ici, néanmoins, la notion de "public» désigne plus une série de réactions qu'un groupe structuré, qui aurait été un écheveau de relations complexes ou qui aurait pu le devenir ${ }^{21}$. Il faut en effet faire une différence entre le "public» conceptuel qu'évoquent la presse et la police, et la praxis sociale. La participation du plus grand nombre n'a aucunement été un réflexe provoqué par un ensemble de stimuli médiatiques - une façon courante de voir les choses, encouragée par la presse et la police. L'appropriation des contenus relève d'un comportement actif et non de la réception passive d'un contenu émis de l'extérieur. Nous ne pouvons néanmoins accéder qu’à la surface de leurs actions, d'autant plus que les acteurs restent difficiles à appréhender : le menuisier des pompes funèbres ou le voisin de pallier restent des figures anonymes pour lesquels, dans la plupart des cas, les données dont nous pouvons disposer se limitent au sexe et à l'âge, parfois à la profession ou au domicile. Les affecter à une classe sociale déterminée n'est pas possible ${ }^{22}$. Ils se différencient toutefois des autres parties prenantes dans les affaires criminelles de deux manières : à l'inverse des experts (scientifiques, journalistes, médecins), ils ne disposent pas de ressources qu'ils pourraient utiliser de manière directe et publique; et à l'inverse des policiers, ils ne disposent pas de jure d'un pouvoir de sanction. Cette recherche procède du constat qu'ils ont pu, malgré tout, avoir une présence médiatique réelle et se voir attribuer des compétences quasi-policières.

pas d'un journal si ce n'était pas le cas; Natural History of the Newspaper, in Everett Cherrington [dir.], Completed Works, vol. 3, Chicago, Illinois University Press, 1951.

21. Cette étude se connecte aux résultats de différents travaux théoriques, qu'il s'agisse d'histoire de la presse ou d'anthropologie historique : Alexander Kluge et Oskar Negt, Öffentlichkeit und Erfahrung. Zur Organisationsanalyse von bürgerlicher und proletarischer Öfentlichkeit, Frankfurt-amMain, Suhrkamp, 1972; Michel de Certeau, Kunst des Handelns, Berlin, Merve, 1988; John Fiske, Reading Popular Culture, Londres, Routledge, 1989; Thomas Lindenberger, Straßenpolitik. Zur Sozialgeschichte der politischen Ordnung in Berlin 1900-1914, Berlin, Dietz Verl., 1992; Alf Lüdtke, "Stofflichkeit, Macht-Lust und Reiz der Oberflächen. Zu den Perspektiven von Alltagsgeschichte», in Winfried Schulze [dir.], Sozialgeschichte, Alltagsgeschichte, Mikro-Historie, Göttingen, 1994, p. 65-80; Dominique Kalifa, L'encre et le sang, op. cit.; Kaspar Maase, «Kinder als Fremde, Kinder als Feinde. Halbwüchsige, Massenkultur und Erwachsene im wilhelminischen Kaiserreich ", Historische Anthropologie, 4, 1996, p. 93-126; Bernd Sösemann, «Einführende Bemerkungen zur Erforschung der Geschichte der Medien und der öffentlichen Kommunikation in Preußen », in id. [dir.], Kommunikation und Medien in Preußen vom 16. bis zum 19. Jahrhundert, Stuttgart, Steiner, 2002, p. 9-21; Achim Landwehr, «Kulturgeschichte des Politischen. Diskurs-Macht-Wissen. Perspektiven einer Kulturgeschichte des Politischen", Archiv für Kulturgeschichte, 85, 2003, p. 71-117; Fabio Crivellari et Marcus Sandl, «Die Medialität der Geschichte. Forschungsstand und Perspektiven einer interdisziplinären Zusammenarbeit von Geschichts- und Medienwissenschaften ", Historische Zeitschrift, 277, 2003, p. 619-654.

22. Cf. pour plus de détails, sur l'affaire Henning, Philipp Müller, Suche nach dem Täter, op. cit., p. 266-277, et 380-384. 


\section{MÉdiatisation DU CRIME \\ ET POLITIQUE DE COMMUNICATION POLICIÈRE}

Le Berliner Lokal-Anzeiger, d'obédience gouvernementale, n'est pas le seul organe de presse à s'emparer de la découverte du cadavre de Lucie Berlin le 11 juin 1904. Les journaux libéraux concurrents, comme le Berliner Tageblatt et le Berliner Morgenpost, les titres à fort tirage, tout comme l'ensemble des autres organes de presse de la capitale sont également informés des derniers résultats de l'enquête criminelle par un communiqué envoyé directement par les autorités policières ${ }^{23}$. C'est déjà là un procédé de routine, qui procède d'une politique de communication de la part de la police : le $6^{\text {e bureau de }}$ la préfecture de police de Berlin est chargé de ces envois, qu'il s'agisse de l'effraction d'un "mauvais garçon" dans un magasin d'articles coloniaux, de l'arrestation du coursier Ewald Krause, qui avait détourné 800 marks, ou encore de la découverte du cadavre de la petite $\mathrm{Lucie}^{24}$. Au début du mois déjà, on avait trouvé un premier cadavre à Charlottenburg, et la police avait mené une campagne d'information similaire ${ }^{25}$. Deux fois par jour, afin de fournir de manière équitable les journaux du matin et ceux du soir, la police envoyait donc aux différentes rédactions des éléments d'enquête parfois très détaillés, au gré de l'avancée des poursuites criminelles en cours : communiqués, déclarations des témoins, des suspects ou des dénonciateurs, résultats de perquisitions, détails sur le mode de vie et le milieu fréquenté par la victime, signalement du coupable présumé, et parfois même images de l'arme du crime ou photographies des criminels recherchés ou arrêtés ${ }^{26}$.

Ces habitudes de communication de la police expliquent en grande partie la présence médiatique régulière des crimes dans la presse locale. Les rédactions se réjouissent de cette ouverture de la police aux médias, contrastant avec la «fermeture bureaucratique» souvent décriée des administrations prussiennes $^{27}$. En somme, si la vie de la métropole est devenue la principale story de la presse de masse, la police y a joué un rôle certain ${ }^{28}$. Le Berliner Tage-

23. $B L A, 11.6 .1904, \mathrm{n}^{\circ} 270$, p. 1. Le Berliner Morgenpost ne fit paraître la nouvelle que dans l'édition du lendemain, en raison de sa politique de périodicité qui ne prévoyait qu'une édition par jour. La rédaction a néanmoins rattrapé son retard en faisant placarder des affiches, $B M P, 12.6 .1904$, $n^{\circ}$ 136, supplément, p. 1. Cf. Philipp Müller, Suche nach dem Täter, op. cit., p. 143-148; cf. aussi, Dominique Kalifa et Marie-Eve Therenty, "Ordonner l'information », in Dominique Kalifa, Philippe Régnier, Marie-Ève Therenty et Alain Vaillant [dir.], La Civilisation du journal. Histoire culturelle et littéraire de la presse française, Paris, Nouveau Monde éd., 2011, p. 897 et sq.

24. LAB A Pr. Br. Rep. 030 Tit. 28 Nr. 806 ministre de l'Intérieur à préfecture de la Police 12.3.1885, Bl. 342; cf. également BLA 23.10.1896, n 500, p. 2; Philipp Müller, Suche nach dem Täter, op. cit., p. 98-140; Dominique Kalifa fait des observations similaires, L'encre et le sang, op. cit., p. 44 et $s q$.

25. BMP, 21.6.1906, $\mathrm{n}^{\circ} 143,1^{\text {er }}$ supplément, p. 1.

26. $B T, 24.8 .1891, \mathrm{n}^{\circ} 426$, et $26.8 .1891, \mathrm{n}^{\circ} 429$.

27. LAB A Pr. Br. Rep. 030 Tit. 94 Nr. 12589, coupure du BT 14.8.1878, Bl. 33.

28. Rolf Lindner, Die Entdeckung der Stadtkultur. Soziologie aus der Erfahrung der Reportage, Frankfurt-am-Main, Campus, 1990, p. 19. 
blatt, pourtant situé au pôle libéral de l'échiquier politique, loue ainsi le fait que «les représentants de la presse qui se présentent dans les bureaux de la préfecture de police - sans que soit faite de différence relative à leur positionnement partisan - se voient donner des éléments d'information, de manière diligente, au sujet de tous les événements criminels importants, et notamment lorsque la participation de la presse paraît souhaitable ${ }^{29}$.

La presse locale se procure ainsi à bon compte des informations intéressantes et aisément renouvelées; elle cherche par ailleurs des nouvelles de nature "atypique, inattendue, qui sortent des sentiers battus " ${ }^{30}$. Les récits policiers sont alors pain bénit.

À l'inverse, la police criminelle se fait une place dans un paysage médiatique en mutation ${ }^{31}$. Depuis la fondation de l'Empire allemand s'étaient développés à Berlin des journaux qui transformaient des événements locaux en affaires d'intérêt général - suivant en cela le modèle des journaux de diffusion nationale et aussi l'exemple de certains de leurs homologues étrangers. Au-delà des oppositions idéologiques et sociales, tous les journaux en question et leur clientèle d'abonnés s'intéressent en premier lieu à la nouvelle capitale. Dans sa première édition, le Berliner Tageblatt insiste sur les liens qui l'unissent à la capitale et légitime son existence par le changement de statut politique de l'ancienne capitale royale à la suite de l'unité allemande. Il consacre aux affaires locales un nombre de pages inhabituellement élevé pour l'époque, habitude imitée par le Berliner Lokal-Anzeiger fondé douze ans plus tard, avec la rédaction locale la plus nombreuse de la ville : sept rédacteurs assignés aux nouvelles locales par le directeur de la publication, August Scherl, qui en outre engage des détectives, des reporters et des intervieweurs ${ }^{32}$. Dans le cas des affaires criminelles, ces dépenses s'avèrent superflues puisque les communiqués de la police sont gratuits et plus fiables ${ }^{33}$.

29. $B T, 30.9 .1891, \mathrm{n}^{\circ} 494$, supplément, p. 1.

30. Rolf Lindner, Die Entdeckung der Stadtkultur, op. cit., p. 17; Niklas Luhmann, Die Realität der Massenmedien, Opladen, Westdt. Verl., 1985, p. 27.

31. Philipp Müller, Auf der Suche nach dem Täter, op. cit., p. 33-92; cf. également Martin Kohlrausch, Monarch im Skandal. Die Logik der Massenmedien und die Transformation der wilhelminischen Monarchie, Berlin, Akad. Verl., 2005; Frank Bösch, Öffentliche Geheimnisse. Skandale, Politik und Medien in Deutschland und Großbritannien 1880-1914, München, Oldenbourg, 2009.

32. Joachim Klippel, Geschichte des Berliner Tageblatts von 1872 bis 1880, Dresden, Risse Verl., 1935, p. 60 ; Thomas Enke, «Die Presse Berlins in der Statistik des Königlichen Polizeipräsidiums (II). Eine Bestandsaufnahme zur Entwicklung der Tageszeitungen in der Reichshauptstadt zwischen 1878 und 1913-14", Theorie und Praxis des sozialistischen Journalismus, 16, 1988, p. 40; Rudolf Stöber, «Der 'Berliner Lokal-Anzeiger' und sein Blattmacher Hugo von Kupffer», Publizistik, 39, 1994, p. 323.

33. Andreas Schulz, «Der Aufstieg der 'vierten Gewalt'. Medien, Politik und Öffentlichkeit im Zeitalter der Massenkommunikation", Historische Zeitschrift, 270, 2000, p. 80. 


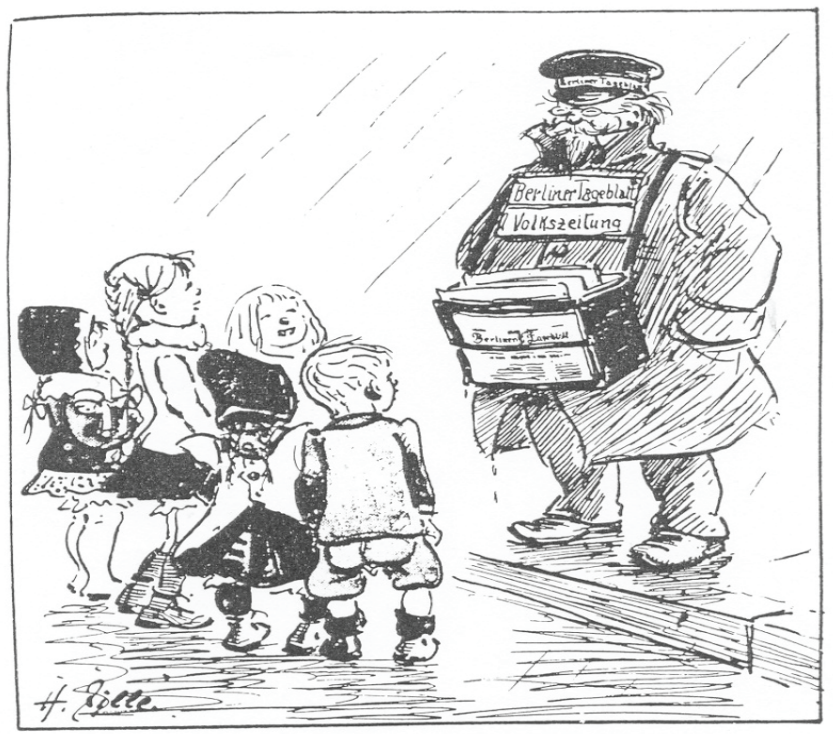

$$
\begin{gathered}
\text { "Sie Zeitumgsoukel, is moih Keen } \\
\text { neier Mord?" }
\end{gathered}
$$

"Dites voir Oncle Journal, alors pas d'nouveau meurtre aujourd'hui?" Caricature de Heinrich Zille (Flüge, Das dicke Zille Buch, Hannover [1914] 1991)

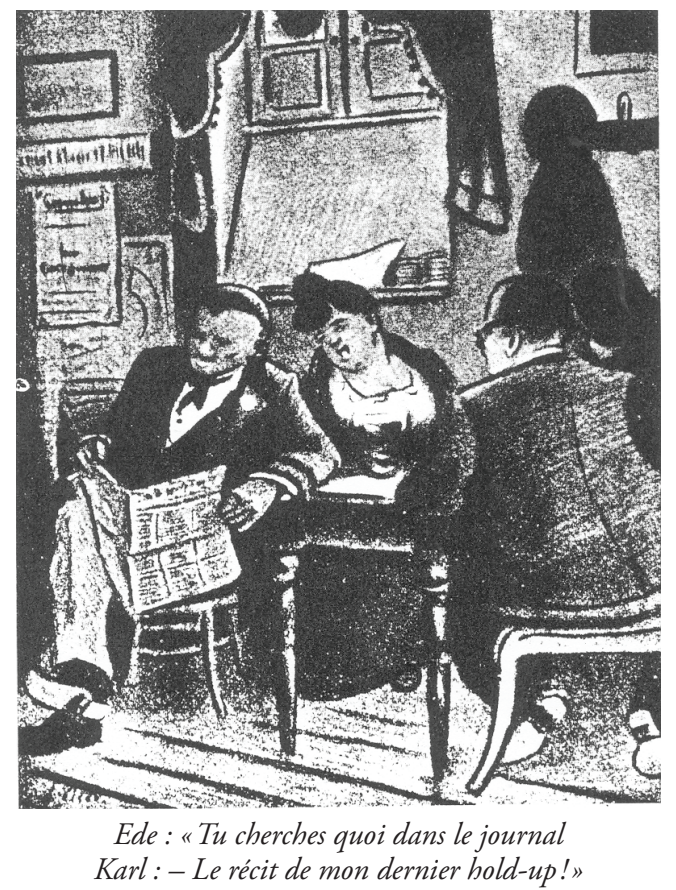

Caricature de Heinrich Zille (Flüge, Das dicke Zille Buch, Hannover [1914] 1991) 
Les informations sur les enquêtes criminelles sont très homogènes dans les différents journaux, jusqu'au style ou aux formulations employées. Les reportages se différencient les uns des autres par d'infimes variantes stylistiques, des raccourcis, des commentaires ou encore par les emplacements des articles dans le journal. Dans l'affaire Lucie Berlin, le Berliner Lokal-Anzeiger lui consacre régulièrement l'article principal de sa première page ${ }^{34}$. Le Berliner Morgenpost, en revanche, place la nouvelle au début de son supplément, tandis que le Berliner Tageblatt ne lui consacre qu'un espace beaucoup plus réduit ${ }^{35}$.

\section{Les communiqués de police: les mutations des années 1880}

La diligence avec laquelle la police de Berlin informait les journalistes au sujet des enquêtes en cours n'avait rien d'évident ${ }^{36}$, tout d'abord en raison de la nécessaire discrétion des enquêtes de police et de la dissimulation des informations, mais aussi parce que les administrations prussiennes avaient à l'époque une conception "étatiste» de l'information administrative conçue comme une propriété de l'Etat qui entraînait une politique ambivalente, positive et offensive à la fois, à l'égard de la presse ${ }^{37}$. Le contrôle s'exerçait par la censure des journaux "indésirables», et par la priorité semi-officielle donnée à certains de leurs concurrents, comme le Berliner Intelligenzblatt.

Avec la loi sur la presse de 1874 , la censure disparaît, mais non la surveillance administrative des journaux par le ministère de l'Intérieur et par la police $^{38}$. Par la suite, c'est l'influence "positive» qui est privilégiée ${ }^{39}:$ d'où la diffusion de ces communiqués conformes à la vision policière.

34. À la une dans l'édition du soir du $B L A$ du 11.06.1904 ( $\left.{ }^{\circ} 206\right)$, dans les premières pages dans les éditions du 11.6.1904 ( $\left.\mathrm{n}^{\circ} 270\right)$ jusqu’à celle du 22.6.1904 ( $\left.\mathrm{n}^{\circ} 287\right)$.

35. $B M P, 12.6 .1904, \mathrm{n}^{\circ} 136$, jusqu'au 17.6.1904, $\mathrm{n}^{\circ} 140$, ainsi que le 21.6.1904, $\mathrm{n}^{\circ} 143$; dans les deux éditions suivantes en page 2 du supplément.

36. Sur les tensions constantes entre la presse et la police, et sur les tentatives de réguler leurs relations, $c f$. Philipp Müller, Auf der Suche nach dem Täter, cit. op., p. 98 et sq., $c f$. aussi Dominique Kalifa, L'encre et le sang, op. cit., p. 200 et sq.

37. Le contrôle s'étend aux informations publiées par la presse. Publier des articles «qui ne s'en tenaient pas aux faits» d'après la police, entrainait pour le journal un avertissement et celui-ci risquait d'être défavorisé par la suite dans la communication des informations. Cf. Philipp Müller, Auf der Suche nach dem Täter, op. cit., p. 105; "'Educateur' ou 'mauvais garçon'?», loc. cit., p. 96; cf. en général Jörg Requate, Journalismus als Beruf. Entstehung und Entwikclung des Journalistenberufs im 19. Jahrhundert. Deutschland im internationalen Vergelich, Göttingen, Vandenhoeck\&Ruprecht, 1995, p. 328 , p. 393 et 395.

38. Cette surveillance englobe les données concernant les tirages, la tendance politique du journal, et les infractions pénales : GStA PK PK I. HA Rep. 77 Tit 54 a Nr. 19 Bd. 2-5, Répertoires de la presse 1903-1913. La police conservait par ailleurs des collections de coupures de presse sur les affaires criminelles, par exemple le meurtre Sinnig LAB A Pr. Br. Rep 030 Tit. 198B Nr. 1688, $c f$. Philipp Müller, Auf der Suche nach dem Täter, op. cit., p. 95.

39. Jörg Requate, Journalismus als Beruf, op. cit., p. 244 et sq.; Rudolf Stöber, Deutsche Pressegeschichte. Von den Anfängen bis zur Gegenwart, Konstanz, 2005, p. 129 et sq. 


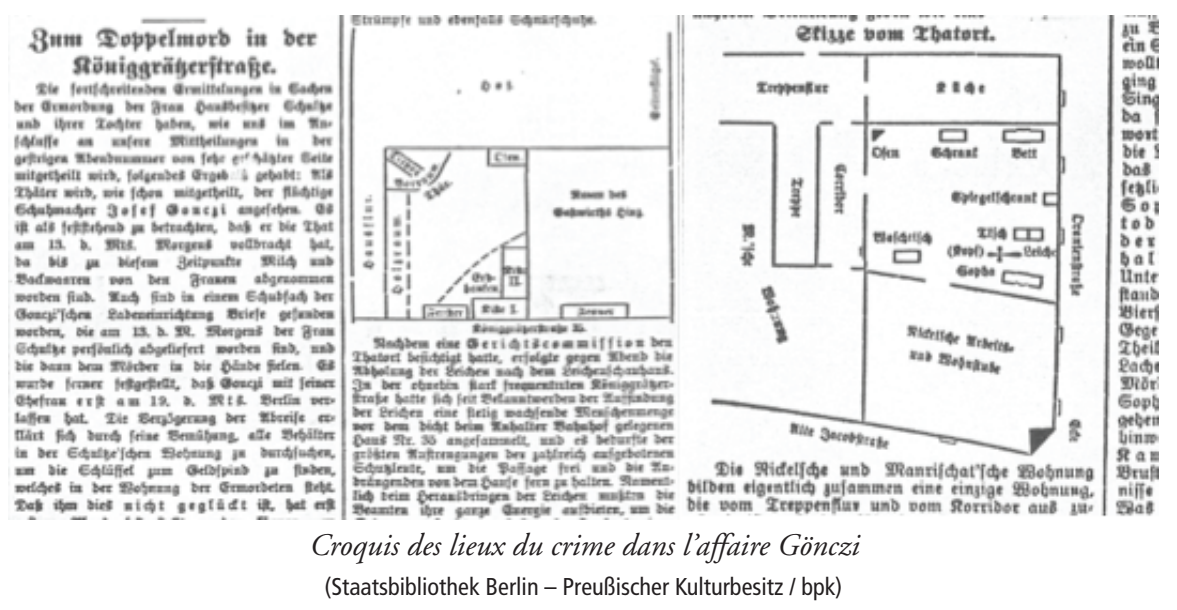

C'est en 1884 que la police criminelle commence à alimenter les journaux locaux en informations semi-officielles, cessant de réserver les informations internes à des réseaux de confiance. Dès lors, la liste des «organes d'importance locale $»^{40}$ incorpore des journaux qui, quelques années auparavant, étaient encore listés dans les «feuilles à scandale» ou "de libre-pensée", voire comme "démocrate, judaïque et progressiste-extrémiste»" ${ }^{41}$, comme le Berliner Zeitung.

Cette décision provient d'abord d'un calcul politique, lié à la logique médiatique des tirages. Les tirages des journaux considérés comme conservateurs par la police, comme le Berliner Fremdenblatt (9500 exemplaires), le Reichsbote (12300), ou encore le Norddeutsche Allgemeine Zeitung (7500) n'étaient pas négligeables, mais cependant nettement inférieurs à ceux de la presse libérale. Ceux de la presse estampillée comme "progressiste», comme le Berliner Tageblatt (60000 exemplaires), qui est dans les années 1880 le journal-phare de la ville, le Berliner Börsen-Courier (10000), ou encore la «feuille à scandale judéo-progressiste» qu'est censé être le Berliner Zeitung (17 500), en faisaient les journaux les plus diffusés en ville. C’est pourquoi, dans ses notes de service, le directeur de la Kriminalpolizei recommande à ses supérieurs de ne pas renseigner uniquement les journaux conservateurs, et que ses conseils sont entendus : «moins lus, [ces communiqués] manqueraient leur cible ${ }^{42}$.

40. LAB A Pr. Br. Rep. 030 Tit. 28 Nr. 806, Rapport du $4^{e}$ bureau à la préfecture de police du 25.6.1885, p. 329.

41. GStA PK I. HA Rep. 77 Tit. 54 Nr. 19 Bd. 3 V 1899, cf. Müller, Auf der Suche nach dem Täter, op. cit. p. 380.

42. Ibidem. 
Les enquêtes criminelles dans une métropole : les "sensations" semi-officielles

Le but de ces $"$ notes de presse ${ }^{43}$ envoyées par la police est la diffusion de l'information, au sens le plus large du terme. Il s'agit de mettre au grand jour le travail accompli par la police, pour donner une résonance publique à ses résultats et à ses succès et mettre un frein à la "critique haineuse et malveillante» dont celle-ci serait victime ${ }^{44}$. Dans les cas de crimes graves, la police a l'ambition d'orienter la perception des enquêtes : «la publication des matériaux primaires de l'enquête permet, lors des crimes graves, d'éviter que le public ne soit submergé d'informations plus ou moins fiables, collectés par les journalistes en utilisant leurs propres moyens - ce qui conduirait également, potentiellement, à perturber les enquêtes ${ }^{45}$.

Parallèlement aux avis de recherche placardés sur les colonnes Morris et à l'affichage dans les bâtiments de la préfecture de police ${ }^{46}$, à partir de 1907 les éditions spéciales du Berliner Morgenpost et du Berliner Lokal-Anzeiger apparaissent comme un vecteur central des enquêtes ${ }^{47}$ qui privilégient donc les quotidiens.

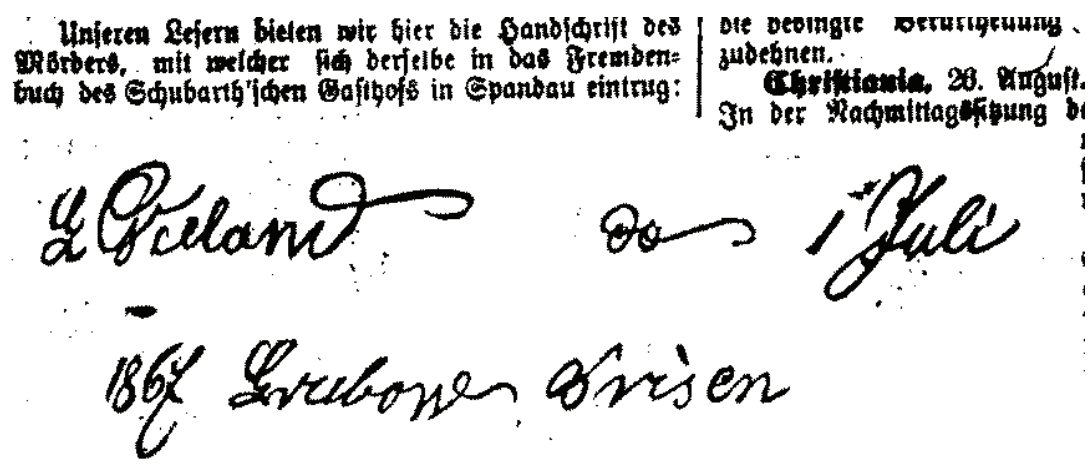

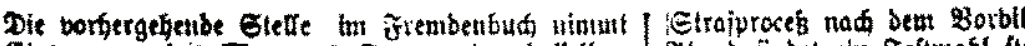

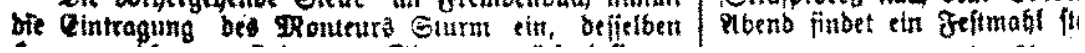
La signature de Wetzel

(Staatsbibliothek Berlin - Preußischer Kulturbesitz / bpk)

L'écriture des criminels en fuite est l'un des éléments criminologiques les plus utilisés dans ces campagnes de presse. Il en est ainsi dans l'affaire Wetzel : en août 1891, Georg Wetzel s'introduit dans un magasin d'électro-

43. LAB A Pr. Br. Rep. 030 Tit. 28 Nr. 806, Lettre du ministère de l'Intérieur à la préfecture de police, 12.3.1885, p. 342 .

44. GStA PK PK I. HA Rep. 77 Tit. 235 Nr. 1 Bd. 14, Lettre de la préfecture de police au ministère de l'Intérieur, 9.2.1911, p. 4; sur ce point important, $c f$. Müller, "Berlin Jack the Ripper», art. cit., p. 103 sq.

45. GStA PK I. HA Rep. 77 Tit. 235 Nr. 1 Bd. 14, Lettre de la préfecture de police au ministère de l'Intérieur, 8.3.1913, p. 40.

46. LAB A Pr. Br. Rep. 030 Tit. 198B Nr. 1934, note du 23.6.1910 p. 137, note du 14.6.1911 p. 172, note du 23.1.1912, p. 186.

47. LAB A Pr. Br. Rep. 030 Tit. 198B Nr. 1933, note du 2.8.1907, p. 189. 
ménager, et emporte l'argent liquide conservé dans la caisse après avoir tué le commerçant. Durant l'enquête, le Berliner Lokal-Anzeiger attire l'attention sur la signature du criminel en cavale : "Wetzel s'est inscrit dans le registre de l'hôtel Schubert sous le nom de "Wieland", en ornant le "W" majuscule d'une fioriture qu'il utilise habituellement pour écrire son vrai nom. C'est d'autant plus intéressant pour la police que ses vêtements portent également un "W", et qu'il utilise vraisemblablement, en ce moment même, un nom d'emprunt commençant par cette lettre ${ }^{48}$.

Dans son édition suivante, le journal revient sur le thème graphologique et sur l'interprétation des lettres tracées par le suspect, puis deux jours plus tard sur sa signature : "L'écriture du meurtrier est caractéristique. La première moitié du mot "Wieland" trahit sa véritable écriture : ses anciens collègues la connaissent bien - en particulier le "W" ouvragé dont il affecte d'orner son nom. Le reste du mot, en revanche, montre l'effort angoissé qu'a mis le futur criminel à contrefaire son identité ${ }^{49}$.

La signature caractéristique ne permet pas aux seuls agents de police d'identifier le criminel : son habitude de dissimuler son identité derrière des pseudonymes commençant par un «W" orné permet à chaque lecteur d'être en mesure d'identifier le coupable - en particulier les employés des hôtels et des banques, qui peuvent être amenés à être en contact avec lui et à voir son écriture.

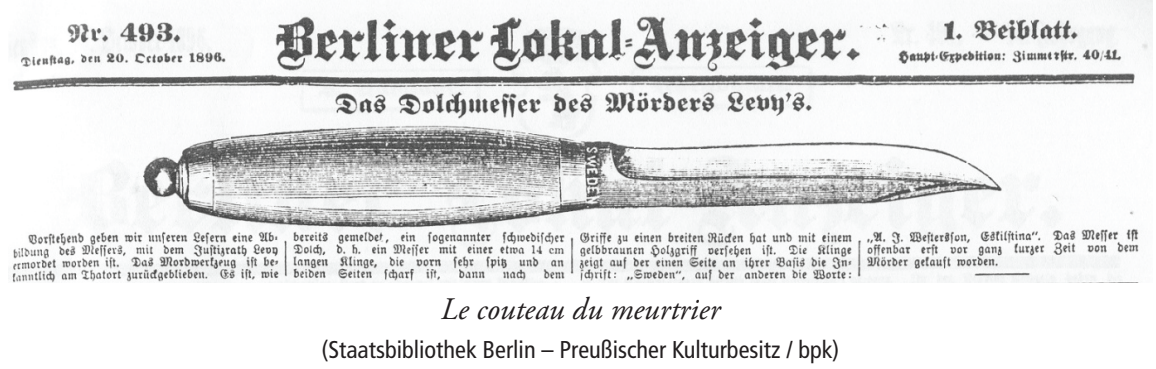

De même lors de l'arrestation de Theodor Berger en juin 1904, alors que les policiers sont persuadés d'avoir appréhendé l'assassin de la petite Lucie Berlin, l'intérêt se porte sur un panier de voyage, de couleur blanche, possession de la prostituée Johanna Liebetruth, la maîtresse de Berger : "Cet objet est très important pour l'enquête : il s'agit d'un panier blanc à couvercle, doté d'une poignée pour le transporter située sur le dessus, et non de deux poignées latérales comme c'est le cas pour les valises plus volumineuses. Il mesure environ 60 centimètres de long et 50 centimètres de haut; c'est le type de panier qu'on peut emmener facilement avec soi, par exemple dans 
le train $"^{50}$. Afin de s'assurer la coopération des gens, on insiste sur l'amnistie et sur la récompense promises à celui qui ramènerait l'objet à la police : "Celui qui le trouvera n’a rien à craindre s'il l'a dissimulé jusque là; il ne sera pas accusé de recel, étant donné que le panier a une valeur inférieure à trois marks. Celui qui le ramènera à la Kriminalpolizei touchera en revanche une part notable de la somme promise pour l'élucidation de l'affaire ${ }^{51}$.

\section{L'engagement du public : un opportunisme obstiné}

Les informations publiées dans les journaux dans ces affaires ne manquent pas leur cible. À la suite des avis de recherche lancés dans le Berliner LokalAnzeiger contre le cambrioleur-meurtrier Bruno Werner ${ }^{52}$, trois habitants de Neumühle près de Königs-Wusterhausen font savoir à la police qu'ils auraient vu le coupable présumé : «Un jeune homme portant des vêtements bon-marché est apparu entre sept heures et sept heures et demie au restaurant "Altenkrug". Il est entré dans la salle en restant là, debout, avec un air ahuri, en gardant sans chapeau sur la tête. Peut-être a-t-il cru avoir identifié un policier en civil parmi les trois personnes présentes. A une question posée par l'un des clients, il a répondu d'un air gêné en demandant si l'on avait vu une femme avec laquelle il avait rendez-vous, puis il est parti. Le jeudi suivant est arrivé le numéro du Berliner Lokal-Anzeiger de la veille, avec le portrait du criminel. Les trois messieurs qui l'avaient vu au restaurant l'ont immédiatement reconnu ${ }^{53}$.

L'enquête Lucie Berlin montre des mécanismes semblables. Dès la découverte du corps, la Kriminalpolizei se met à la recherche du malfaiteur, et le $4^{\mathrm{e}}$ bureau en appelle aussitôt aux témoignages des lecteurs de la presse, en révélant que la jeune fille, le jour du meurtre, s'était rendue au n ${ }^{\circ} 130$ de la Ackerstraße pour écouter un orgue de barbarie avec ses amies. Alors que les autres enfants avaient suivi l'artiste dans la cour de l'immeuble voisin, elle était restée sous le porche en compagnie de deux jeunes hommes ${ }^{54}$. La police voit immédiatement en eux les auteurs du "crime atroce» du nord de la ville. Le suspect principal serait «un homme d'environ 20 ans, avec un paletot gris-clair et un chapeau blanc en tissu " 55 . Dans les éditions suivantes, les journaux se font l'écho des détails concernant ces individus «fortement suspects " ${ }^{56}$, complétés dans les éditions du soir par une description exhaustive de leur apparence.

50. BMP, 19.6.1904, $\mathrm{n}^{\circ} 142,1^{\text {er }}$ supplément, p. $3 ; B L A, 19.6 .1904, \mathrm{n}^{\circ} 282$, p. 3.

51. $B L A, 19.6 .1904, \mathrm{n}^{\circ} 282$, p. $3 ; B M P, 19.6 .1904, \mathrm{n}^{\circ} 142,1^{\text {er }}$ supplément, p. 3.

52. $B L A, 22.10 .1896, \mathrm{n}^{\circ} 498$, p. 2 ; $c f$. aussi $B L A, 26.10 .1896, \mathrm{n}^{\circ} 504$, p. 1 .

53. $B L A, 24.10 .1896, \mathrm{n}^{\circ} 501$, p. 1 .

54. $B L A, 11.6 .1904, \mathrm{n}^{\circ} 270$, p. $1 ; B L A, 12.6 .1904, \mathrm{n}^{\circ} 271$, p. $2 ; B L A, 12.6 .1904, \mathrm{n}^{\circ} 273$; $B M P, 14.6 .1904, \mathrm{n}^{\circ} 137,1^{\text {er }}$ supplément, p. 1.

55. $B L A, 11.6 .1904, \mathrm{n}^{\circ} 270$, p. $1 ; B L A, 12.6 .1904, \mathrm{n}^{\circ} 294,1^{\text {er }}$ supplément, p. 1 .

56. $B L A, 12.6 .1904, \mathrm{n}^{\circ} 271$, p. 3 . 
Le 12 juin, un certain Otto Lenz est identifié comme étant l'homme en question, et emprisonnéén ${ }^{57}$. Au préalable, des voisins, des passants et des relations des policiers, avaient confirmé la chose par des déclarations qui avaient jeté la suspicion sur le prévenu. La police apprend ainsi qu'il avait habité au 130 de la Ackerstraße jusqu'à peu de temps auparavant, et qu'il avait récemment changé de domicile. D'après les voisins, il aurait maintenu par la suite le contact avec la victime, et donné "des leçons de danse dans les règles de l'art» à la fille des voisins ${ }^{58}$. Un cocher fait savoir à la police, qu'il avait observé, depuis le croisement de la Feldstraße et de la Ackerstraße, «Lenz et la jeune fille dans un square, où il les a vu arriver et avait remarqué que Lenz s'asseyait sur un banc avec elle et qu'ils mangeaient des bonbons ensemble " ${ }^{59}$. Un autre témoin signale de son côté qu'il «travaillait à sa fenêtre le jeudi fatidique, comme à son habitude, alors que l'organiste jouait et que les enfants dansaient; il avait alors vu Lenz qui se tenait sous le porche " $"$. Aussi lourds que soient ces soupçons contre Otto Lenz, ils apparaissent cependant peu à peu comme des hypothèses et de vaines spéculations, lorsque la police arrête le souteneur Theodor Berger, lui aussi domicilié au 130 de la Ackerstraße.

Le public est ici intégrée au processus de l'enquête. L'appel à «apporter sa contribution à l'arrestation du suspect ${ }^{61}$ " n'est pas seulement verbal, mais il est bien suivi d'effet. L'arrestation d'Otto Lenz le 12 juin résulte ainsi de l'intervention d'un de ces éléments du "public»: «L'une de ses connaissances a croisé Lenz dans un bistrot, qu'il ne fréquentait pas auparavant, et lui dit qu'il était celui que la police criminelle recherchait. Lenz n'a rien voulu savoir. L'autre est resté sur son affirmation que la description correspondait entièrement, et lui a laissé le choix de se rendre seul au commissariat, sans quoi il l'y amènerait. Lenz s'est finalement décidé à s'y rendre, accompagné par cette connaissance ${ }^{62}$.

Mettre en demeure de parler, confronter le signalement et la personne physique, confondre le suspect et exercer une pression immédiate : les méthodes employées ici sont des procédés spécifiquement policiers, que l'inconnu s'approprie.

Les "arrestations spontanées" par des civils ne se déroulent cependant pas toujours de manière facile. Elles supposent un engagement physique immédiat, et donc pour l'individu bénévole d'être disposé à employer la violence envers une autre personne, et de prendre le risque d'être soi-même blessé.

57. $B L A, 12.6 .1904, \mathrm{n}^{\circ} 272$, p. $2 ; B T, 13.6 .1904, \mathrm{n}^{\circ} 296$, p. $4 ; B L A, 13.6 .1904, \mathrm{n}^{\circ} 273$, p. 3 ; $B M P, 14.6 .1904, \mathrm{n}^{\circ} 137,1^{\text {er }}$ supplément, p. 1.

58. $B L A, 14.06 .1904, \mathrm{n}^{\circ} 275$, p. $2 ; B M P, 15.06 .1904, \mathrm{n}^{\circ} 138,1^{\text {er }}$ supplément, p. $1, B L A$, 17.6.1904, nं 279 , p. 3.

59. $B L A, 15.06 .1904, \mathrm{n}^{\circ} 275$, p. $3 ; B T, 15.6 .1904, \mathrm{n}^{\circ} 299,1^{\text {er }}$ supplément, p. $1 ; B M P$, 16.6.1904, $\mathrm{n}^{\circ} 139,1^{\text {er }}$ supplément, p. 1.

60. $B L A, 15.6 .1904, \mathrm{n}^{\circ} 275$, p. $3 ; B T, 15.6 .1904, \mathrm{n}^{\circ} 299,1^{\text {er }}$ supplément, p. 1.

61. $B L A, 29.8 .1891, \mathrm{n}^{\circ} 401$, p. 3 .

62. $B L A, 14.6 .1904, \mathrm{n}^{\circ} 275$, p. $2 ; B T, 14.6 .1904, \mathrm{n}^{\circ} 298$, p. 4 ; BMP, $15.6 .1904, \mathrm{n}^{\circ} 138,1^{\text {er }}$ supplément, p. 1. 
L'arrestation spectaculaire d'Otto Lenz atteste l'atmosphère émotionnelle extrêmement lourde qui règne dans les environs du lieu du crime, les jours qui suivent celui-ci. La nouvelle se répand par la suite « rapidement dans tout le nord de la ville, et a suscité une grande effervescence dans la Ackerstraße. Un autre individu a d'ailleurs été pris à parti dans la rue, accusé du meurtre à son tour, et menacé de se faire passer à tabac, d'autant plus qu'il a pris peur et s'est enfui. La police a dû le mettre à l'abri des débordements et contenir la fureur populaire $»^{63}$.

Poursuivre, appréhender, livrer et le cas échéant punir soi-même le coupable présumé sont des éléments qui reviennent de manière récurrente dans ces chasses aux criminels. Par ailleurs, les observations ou les signalements préalables aux arrestations se distinguent par des combinaisons mentales propres aux individus, et qui n'ont pas grand-chose à voir avec un raisonnement criminologique. C'est ainsi qu'en août 1891, durant l'affaire Wetzel, un groupe d'ouvriers amènent à un gendarme un jeune homme qui s'était rendu suspect à leurs yeux par un comportement qui ressemblait à une fuite. Ce dernier s'était «joint à eux et ils ont commencé à converser. L'inconnu a pris la fuite au moment où le sujet du meurtre commis a été abordé - les ouvriers l'ont cependant attrapé et amené à la gendarmerie „ ${ }^{64}$. Trois jours plus tard, des passants ont d'autres raisons encore de tenter d'arrêter un hypothétique Wetzel :

"Vers 11 heures, plusieurs personnes se trouvaient devant la colonne Morris à l'angle de la Königsstraße et de la Jüdenstraße, et regardaient attentivement le portrait du voleur-meurtrier W. sur l'avis de recherche officiel. Soudain quelqu'un s'est exclamé : "Là! C'est Wetzel!" - et en effet, un homme se tenait là, qui bavardait avec une dame devant l'affiche, et dont les traits ressemblaient à s'y méprendre au visage du suspect. Les gens se sont aussitôt jetés sur ce monsieur, qui s'est mis à crier et à protester qu'il n'était pas Wetzel. Il a alors été mis brutalement en demeure de montrer ses papiers $"$ " plutôt la règle que l'exception ${ }^{66}$.

La mise en œuvre des recherches n'a pas de lieu spécifique ou privilégié dans une métropole, dans la mesure où le malfaiteur peut y être croisé partout. Que ce soit au bistrot, dans la forêt, dans la rue ou dans le métro, chacun y trouve une occasion d' "en être" et de participer. ${ }^{67}$ Ces affaires locales

63. $B T, 14.6 .1904, \mathrm{n}^{\circ} 298$, p. 4 .

64. $B L A, 27.8 .1891, \mathrm{n}^{\circ} 397$, p. 3.

65. $B L A, 30.8 .1891, \mathrm{n}^{\circ} 403$, p. 3.

66. $B L A, 15.8 .1905, \mathrm{n}^{\circ} 398$, p. $2 ; B L A, 12.8 .1905, \mathrm{n}^{\circ} 394$, p. $2 ; B M P, 10.2 .1905, \mathrm{n}^{\circ} 35 ; c f$. aussi Lindenberger, Straßenpolitik, op. cit., p. 133-136.

67. J'ai fait le choix de renoncer ici à développer la notion de moral panic, telle qu'elle est utilisée par exemple par Herbert Reinke, Richard Evans ainsi que Benjamin C. Hett pour des événements médiatiques comparables à l'époque de l'Empire - cf. notamment Herbert Reinke, “'Armed as if for a war.' The state, the military and the professionalization of the Prussian Police in Imperial Germany", in Clive Emsley et Barbara Weinberger [dir.], Policing in Western Europe 1850-1940. Politics, professionalization and public order, New York, Greenwood Press, 1991, p. 62; Richard J. 
touchent les habitants dans leur univers de vie (Lebenswelt) : ces recherches les concernent et ils veulent les mener à bien de manière active - ce qui explique la manière systématique avec laquelle la population urbaine prête la main aux enquêtes policières. Plus encore : ils interviennent de manière publique, afin de se saisir du meurtrier, et mettent leur jugement et leur appréciation personnels au service de la coopération avec la police ${ }^{68}$. Des signes extérieurs de peur ou d'angoisse, une ressemblance fortuite avec la physionomie du suspect, un nom voisin ${ }^{69}$, une situation de précarité financière visible ${ }^{70}$, sont des occasions de susciter des soupçons et entraînent des conséquences pour les personnes concernées.

Face au cas de Lucie Berlin, le Berliner Beobachter se complaît à dénoncer ce qu'il appelle «le sensationnel " ${ }^{71}$, défini comme l'ensemble des aspects "cruels, mystérieusement fascinants, bref, criminels» de l'affaire. Les commentaires lui opposent des "motifs plus purs et plus nobles": "c'est la pitié, la commisération intime et qui vient du cœur, envers le destin tragique de cette malheureuse enfant abattue comme un animal, et envers la douleur atroce de ses parents qui sont bien à plaindre $»^{72}$. Le journal, de sensibilité gouvernementale, montre une contradiction entre ces remarques moralisantes et ses priorités concernant la transmission des informations, vu qu'il consacre une part importante de ses numéros aux communiqués de police et aux appels au public. Dans la réalité de ce que publie le journal, la recherche du sensationnel se conjugue avec l'expression de la pitié : la collecte de fonds lancée par les voisins, le geste commercial des pompes funèbres font partie de ces "sentiments de participation ${ }^{73}$ fondés sur la pitié mais la colère envers les coupables supposés n'en apparaît pas moins comme l'autre forme de ces réactions. Lorsque la police emmène Lenz puis Berger voir le corps de Lucie Berlin, les deux prévenus se font ainsi accueillir devant la morgue par la foule qui les couvre de jurons et d'insultes ${ }^{74}$. De telles informations sont aussi publiées dans le cadre de l'affaire Wetzel. Tandis que des passants «en extrêmement grand nombre» menacent et insultent le prévenu lors de son transfert à la prison de Spandau, des badauds qui venaient pour la kermesse de

Evans, Tales from the German Underworld. Crime and Punishment in the Nineteenth Century, New Haven, Yale University Press, 1998, p. 192; Benjamin C. Hett, Death in the Tiergarten. op. cit., 2004, p. 78. Forgé par Stanley Cohen dans Folk Devils and Moral Panics, Londres, MacGibbon \& Kee, 1972, puis popularisé récemment par David Garland dans "On the concept of moral panic", Crime Media Culture, 4, 2008, p. 9-30), le terme de moral panic s'avère problématique car il fait peser sur les comportements des acteurs des appréciations disqualifiantes ou normatives (l'irrationalité, l'inadaptation) peu conformes avec la méthode historique.

68. Ces phénomènes ne débouchent cependant pas sur davantage de discipline de la part du "public» : cf. Vanessa Schwartz, Spectacular Realities. Early Mass Culture in Fin-de-Siècle Paris, Berkeley, University of California Press, 1998, p. 5 et $s q$.

69. $B L A, 21.10 .1896, \mathrm{n}^{\circ} 496$, p. 1.

70. $B L A, 30.10 .1896, \mathrm{n}^{\circ} 511$, p. 1 et $s q$.

71. $B L A, 19.6 .1904, \mathrm{n}^{\circ} 283,1^{\text {er }}$ supplément, p. 1.

72. Ibidem.

73. $B L A, 19.6 .1904, \mathrm{n}^{\circ} 283,1^{\text {er }}$ supplément, p. 1.

74. $B L A, 17.6 .1904, \mathrm{n}^{\circ} 273$, p. 3; sur Grosse et Werner, $B L A, 27.10 .1896, \mathrm{n}^{\circ} 506$, p. 2. 
l'année vont visiter la maison de la victime sur la Breitenstraße $\mathrm{e}^{75}$. La nature des crimes en question explique le déchaînement de la violence verbale, voire physique, envers les malfaiteurs. Dans le cas de Lucie Berlin, les découvertes des enquêtes alourdissent encore la faute du coupable dans la perception du public : elle a en effet été d'abord violée, puis étranglée, et l'"horreur» de ce crime sexuel s'impose face à la représentation d'une enfant de neuf ans présentée dans la presse comme "une pauvre petite créature sans défense», et comme une jeune fille extrêmement joyeuse et éveillée ${ }^{76}$.

\section{LA DisCipline DU COMMENTAIRE : OBSERVATIONS SOCIAL-HYGIÉNISTES SUR LE «SENSATIONNEL »}

Alors que la presse locale manifeste son "effroi» face à ces "crimes sanglants $»^{77}$, ses organes comme le Berliner Lokal-Anzeiger ou le Berliner Morgenpost ne se privent pas d'aiguillonner la colère publique. La police criminelle constate par exemple, lorsqu'elle inspecte le quartier où habite Lucie Berlin, que s'y côtoient sans séparation nette des gens "honnêtes" et "malhonnêtes». Dans le voisinage immédiat, les relations entre les uns et les autres se passent de manière insouciante et naturelle. Pourtant, tandis que la famille Berlin jouit de "la meilleure réputation possible», la condition matérielle d'Otto Lenz et de Theodor Berger repose sur la prostitution de leurs compagnes respectives, Seiler et Liebetruth. La proximité confiante dont attestent les attentions de Lucie pour Otto Lenz, leurs conversations ou les «leçons de danse", ne posent apparemment pas problème aux voisins ${ }^{78}$. Le Berliner Beobachter, en revanche, mentionne une "sorte d'indifférence étrange» et l'«ignorance regrettable de risques sérieux" : «les enfants, appartenant aux couches les plus nombreuses de la population, entrent en contact avec des éléments sales et criminels, comme des prostituées ou des souteneurs, qui ne mettent pas seulement en danger leur développement moral, mais les mettent même directement en péril $»^{79}$.

Le fait que des familles respectables aient été au courant des activités suspectes de leurs voisins, et ne s'en soient pas alarmés, est incompréhensible pour les commentateurs. Alors que la proximité désordonnée du «moral» et de l'«immoral» dans cet immeuble constitue le quotidien pour les habitants du 130 Ackerstraße, elle contredit la représentation d'un monde ordonné en fonction de concepts moraux, propres aux classes bourgeoises de l'époque.

75. $B L A, 27.8 .1891, \mathrm{n}^{\circ} 397$, p. 3; $B L A, 31.10 .1896, \mathrm{n}^{\circ} 513$, p. 1 ; il en est de même lors du meurtre de Lucie Berlin, $B M P, 14.6 .1904, \mathrm{n}^{\circ} 137,1^{\text {er }}$ supplément, p. 1.

76. $B L A, 11.6 .1904, \mathrm{n}^{\circ} 270$, p. $1 ; B L A, 13.6 .1904, \mathrm{n}^{\circ} 273$, p. $3 ; B L A, 19.6 .1904, \mathrm{n}^{\circ} 283,1^{\text {er }}$ supplément, p. 1 ; BLA, 12.6.1904, $\mathrm{n}^{\circ}$ 271, p. 3 ; cf. aussi Tanja Hommen, Sittlichkeitsverbrechen. Sexuelle Gewalt im Kaiserreich, Frankfurt-am-Main, Campus, 1999; Kerstin Brückweh, Mordlust. Serienmorde, Gewalt und Emotionen im 20. Jahrhundert, Frankfurt-am-Main, Campus, 2006.

77. $B M P, 12.6 .1904, \mathrm{n}^{\circ} 136,1^{\mathrm{er}}$ supplément, p. 1.

78. $B L A, 11.6 .1904, \mathrm{n}^{\circ} 270$, p. $2 ; B L A, 13.6 .1904, \mathrm{n}^{\circ} 273$, p. 3.

79. $B L A, 19.6 .1904, \mathrm{n}^{\circ} 283,1^{\mathrm{er}}$ supplément, p. 1 . 
On peut faire des observations semblables à propos de l'affaire Grosse et Werner. Ici, ce n'est pas le milieu social qui suscite le rejet, mais le très jeune âge du coupable. Wilhelm Grosse et Bruno Werner ont en effet moins de seize ans lorsqu'ils commettent leur "farce d'adolescents ${ }^{80}$. Quelques jours plus tard, le Berliner Tageblatt titre "Des criminels mineurs" et cite une étude scientifique récente sur la question ${ }^{81}$. Le haut fonctionnaire von Massow commente à dessein l'événement en exhortant à «penser à la jeunesse, car l'État irait à sa décadence et à sa perte si la génération montante dégénérait ${ }^{82}$. Alors qu'on s'efforce de se saisir les criminels en fuite dans les cafés, dans leur voisinage ou dans la rue, quelques-uns se penchent sur la constitution physique de ces jeunes délinquants. Le plus jeune des deux, Wilhelm Grosse, est ainsi présenté comme un "être un peu limité», et comme un "joyeux drille $»^{83}$. Son destin apparaît comme une descente aux enfers, en raison de mauvaises fréquentations qui ont fait d'un garçon auparavant irréprochable un être "négligeant et nonchalant ${ }^{84}$. Cette juxtaposition d'une vie disciplinée passée et d'une dégénérescence présente est visible dans la biographie qu'il a dû écrire à la préfecture de police et que les journaux commentent : «Le style de ce qu'il a écrit est maladroit et l'orthographe [incertaine] montre que la personne qui écrit est sur le pied de guerre; son écriture est cependant surprenante, dans la mesure où elle s'étale joliment et de manière élégante ${ }^{85}$.

Son complice Bruno Werner n'est pas davantage épargné, en matière de remarques pathologisantes. Celles-ci se fondent sur le témoignage des tiers, dans la mesure où Werner est encore en cavale, alors que Grosse a déjà été arrêté au moment où la presse commence à parler de lui. La vie de Werner s'étale néanmoins dans la presse, grâce entre autres à une interview donnée par sa mère : elle y fait état de lésions de la boîte crânienne qui aurait entraîné des troubles durables de la perception, une soif de lecture et une tendance excessive à s'amuser et à se disperser ${ }^{86}$. Un médecin diagnostique de son côté un «terrain criminel [qui aurait] contribué au passage à l'acte», à partir d'une photographie privée du jeune malfaiteur publiée dans les journaux ${ }^{87}$. En se référant aux travaux de Lombroso et d'autres autorités de la criminologie de l'époque, il croit reconnaitre dans la physionomie de Bruno Werner : «maints signes patents de dégénérescence, comme on en trouve fréquemment dans les cas de criminels récidivistes. Parmi ceux-ci les oreilles décollées, le crâne

80. $B L A, 21.10 .1896, \mathrm{n}^{\circ} 495$, p. 1.

81. BLA, 24.10.1896, $\mathrm{n}^{\circ} 501$, p. 1 sq., ici p. 2; Lino Ferriani, Minderjährige Verbrecher. Versuch einer strafgeschichtlichen Psychologie, Berlin, 1896.

82. BLA, 24.10.1896, n ${ }^{\circ} 501$, p. 2.

83. BLA, 21.10.1896, n० 495 , p. 1.

84. $B T, 21.10 .1896, \mathrm{n}^{\circ} 537,1^{\text {er }}$ supplément, p. 1.

85. BLA, 22.10.1896, n ${ }^{\circ} 498$, p. 2.

86. BLA, 27.10.1896, n ${ }^{\circ} 506$, p. 2.

87. $B L A, 28.10 .1896, n^{\circ} 507$, p. 2. 
allongé vers le haut et vers l'arrière, la touffe de cheveux gris sur l'occiput, et les yeux qui semblent légèrement bridés ${ }^{88}$.

Cependant, ces réflexions criminologiques ne concernent qu'un groupe restreint de personnes compétentes et le plus grand nombre ne dispose pas des ressources nécessaires. Pour la majorité du public, la chasse au criminel, offre une meilleure opportunité de se faire valoir en public. Mais d'une certaine manière, ces différents modes d'action sont substituables : le fait que l'on ne parvienne pas à arrêter Bruno Werner explique sans doute en partie le déplacement de l'intérêt pour l'endroit où il pourrait se trouver vers sa conformation physique et ses tares éventuelles - une démarche qui permet de l'appréhender en quelque sorte symboliquement, à l'aide des concepts médicaux.

*

La résonance publique et la réception de ces différentes enquêtes sont très variables. Le contexte de chaque situation ainsi que les motivations individuelles y jouent en effet un rôle non négligeable. De même, l'appropriation de ces données par le public s'effectue largement suivant l'arrière-plan social des individus : les appartenances déterminent qui agit et comment, suivant les compétences sociales et culturelles de chacun. Pour beaucoup, la chasse au coupable est une occasion séduisante de sortir de l'anonymat; pour quelques autres, ces affaires récurrentes sont vécues au contraire comme un épisode indésirable, fruit d'une "vie plus intense de la sphère publique ${ }^{89}$. Le rejet du "sensationnel» d'une part, la participation active à celui-ci d'autre part, recouvrent un conflit plus classique ${ }^{90}$, car derrière les déclarations sur le bon ou le mauvais goût des informations transmises par la presse, on retrouve la question des formes et de la répartition des ressources culturelles dans la société. A travers ces cas d'espèce, les acteurs discutent en fait de la légitimité des formes nouvelles et «sensationnelles» que prennent les débats publics. La dénonciation du «sensationnel » permet d'articuler l'agacement vis-à-vis du «nouveau lectorat» et l'incompréhension de ces nouvelles formes de communication publique. Elle témoigne aussi d'une prise de distance par rapport aux mutations en cours dans l'Empire, depuis au moins les années 1880.

Si les habitants s'emparent de cette forme inédite de participation aux affaires publiques de la métropole, c'est aussi en raison d'une conjoncture spé-

88. $B L A, 28.10 .1896, \mathrm{n}^{\circ} 507$, p. 2 ; un troisième élément est une lettre anonyme, $B L A$, $29.10 .1896, n^{\circ} 509$, p. 3 ; cf. Peter Becker, Verderbnis und Entartung. Eine Geschichte der Kriminologie des 19. Jahrhunderts als Diskurs und Praxis, Göttingen, 2002; Richard F. Wetzell, Inventing the Criminal. A History of German Criminology 1880-1945, Chapel Hill, University of North Carolina Press, 2000.

89. $B T, 22.10 .1896, \mathrm{n}^{\circ} 497$, p. 1.

90. Sur l'ambivalence de ces rapports, voir aussi Dominique Kalifa, L'encre et le sang, op. cit., p. 216; Philipp Müller, "Berlin Jack the Ripper", loc. cit., p. 101; Auf der Suche nach dem Täter, op. cit., p. 265. 
cifique : celle d'une mutation du paysage médiatique et du développement tous azimuts de la politique de communication de la police. Ils peuvent alors affirmer leur responsabilité personnelle dans le maintien de la «sécurité» et de l'«ordre». Les communiqués qui rendent public le travail d'élucidation ont permis d'intensifier les relations entre la police et la société, dans la mesure où ils donnaient l'occasion d'agir dans la sphère publique, avec des possibilités plus larges qu'auparavant : désormais, les gouvernés peuvent avoir leur part dans la construction de l'ordre policier. Ces possibilités n'existaient pas auparavant, et elles apparaissent donc pour ainsi dire comme des occasions inconnues de participer à la vie publique, par la parole et par l'action. À travers la collaboration affichée entre la police et la presse, les lecteurs des journaux se trouvent donc valorisés, au-delà de la simple lecture des informations. Par les processus d'appropriation qui sont au cour du média métropolitain qu'est le journal moderne, chacun d'entre eux devient un informateur potentiel, voire un adjuvant des poursuites criminelles. À l'inverse, cette diffusion large marginalise la minorité des «anciens lecteurs" qui perdent leur ancien privilège d'exclusivité, en dépit de compétences beaucoup plus affinées. Ce double visage d'une même évolution montre aussi que les distinctions sociales ont leur porosité : dans l'affaire Grosse et Werner, l'expert médical croise la mère du prévenu lorsqu'il faut donner des témoignages sur sa constitution mentale, et un auteur de lettre anonyme les rejoint. Au-delà des affaires criminelles elles-mêmes, c'est au niveau méta-communicationnel que des correspondances sont visibles. En effet, la chasse au coupable est aussi une forme symbolique ${ }^{91}$ : les protagonistes entrent en contact et échangent, au sujet d'une affaire qui concerne la communauté sociale dans son ensemble. Leur rencontre permet - ne serait-ce que pour un bref moment - de mettre en place une relation sociale et politique entre l'administration, les médias et le public. Le crime «connu de tous parce qu'il est connu ${ }^{92}$, est une référence commune qui tisse des liens discursifs trans-sociaux, tout en maintenant les différences sociales les plus fines. Cet état d'esprit officieux dépasse largement les cas d'espèce sur lesquels enquête la police à un moment donné : il est éminemment créateur d'une "société», puisqu'il pousse les acteurs à une action socialisée et à une réflexion sur celleci; il contribue aussi puissamment au changement social, dans la mesure où il généralise et popularise la «mise en police» (Polizieren) de l'ensemble de la société.

\footnotetext{
Philipp Müller est enseignant-chercheur à la Georg-August Universität de Göttingen
}

91. Robert E. Park, News and the Human Interest Story, op. cit., p. 113.

92. Niklas Luhmann, Die Realität der Massenmedien, op. cit., p. 14; John Fiske, Reading Popular Culture, op. cit., p. 150. 